\title{
FFP3 respirators protect healthcare workers against infection with SARS-CoV-2
}

\author{
Mark Ferris ${ }^{1}$, Rebecca Ferris ${ }^{1}$, Chris Workman ${ }^{1}$, Eoin O'Connor ${ }^{2}$, David A Enoch ${ }^{1,3}$, \\ Emma Goldesgeyme ${ }^{1}$, Natalie Quinnell ${ }^{1}$, Parth Patel ${ }^{1}$, Jo Wright ${ }^{1}$, Geraldine Martell ${ }^{1}$, \\ Christine Moody ${ }^{1}$, Ashley Shaw ${ }^{1}$, Christopher J.R. Illingworth ${ }^{4,5}$, Nicholas J. \\ Matheson ${ }^{1,6,7,8}$, and Michael P. Weekes ${ }^{1,6,9}$ \\ ${ }^{1}$ Cambridge University NHS Hospitals Foundation Trust, Cambridge, UK \\ ${ }^{2}$ School of Clinical Medicine, University of Cambridge, Cambridge, UK \\ ${ }^{3}$ Clinical Microbiology \& Public Health Laboratory, Public Health England, Cambridge, \\ $\mathrm{UK}$ \\ ${ }^{4} \mathrm{MRC}$ Biostatistics Unit, University of Cambridge, East Forvie Building, Forvie Site, \\ Robinson Way, Cambridge, CB2 0SR, UK \\ ${ }^{5}$ Department of Applied Mathematics and Theoretical Physics, University of Cambridge, \\ Cambridge, CB3 0WA, UK \\ ${ }^{6}$ Department of Medicine, University of Cambridge, Cambridge, UK \\ ${ }^{7}$ Cambridge Institute of Therapeutic Immunology and Infectious Disease (CITIID), Jeffrey \\ Cheah Biomedical Centre, University of Cambridge, UK \\ ${ }^{8}$ NHS Blood and Transplant, Cambridge, UK \\ ${ }^{9}$ Cambridge Institute for Medical Research, University of Cambridge, Cambridge, UK
}

June 30, 2021

\section{Introduction}

Consistent with World Health Organization (WHO) advice [1], UK Infection Protection Control guidance recommends that healthcare workers (HCWs) caring for patients with coronavirus disease 2019 (COVID-19) should use fluid resistant surgical masks type IIR (FRSMs) as respiratory protective equipment (RPE), unless aerosol generating procedures (AGPs) are being undertaken or are likely, when a filtering face piece 3 (FFP3) respirator should be used [2]. In a recent update, an FFP3 respirator is recommended if "an unacceptable risk of transmission remains following rigorous application of the hierarchy of control" [3]. Conversely, guidance from the Centers for Disease Control and Prevention (CDC) recommends that HCWs caring for patients with COVID-19 should use an N95 or higher level respirator [4]. WHO guidance suggests that a respirator, such as FFP3, may be used for HCWs in the absence of AGPs if availability or cost is not an issue [1].

A recent systematic review undertaken for PHE concluded that: "patients with SARS-CoV-2 infection who are breathing, talking or coughing generate both respiratory droplets and aerosols, but FRSM (and where required, eye protection) are considered to provide adequate staff protection" [5]. Nevertheless, FFP3 respirators are more effective in preventing aerosol transmission than FRSMs, and observational data suggests that they may improve protection for HCWs [6]. It has therefore been suggested that respirators should be considered as a means of affording the best available protection [7], and some organisations have decided to provide FFP3 (or equivalent) respirators to HCWs caring for COVID-19 patients, despite a lack of mandate from local or national guidelines [8]. 
Data from the HCW testing programme at Cambridge University Hospitals NHS Foundation Trust (CUHNFT) during the first wave of the UK severe acute respiratory syndrome coronavirus 2 (SARS-CoV-2) pandemic indicated a higher incidence of infection amongst HCWs caring for patients with COVID-19, compared with those who did not [9]. Subsequent studies have confirmed this observation [10, 11]. This disparity persisted at CUHNFT in December 2020, despite control measures consistent with PHE guidance and audits indicating good compliance. The CUHNFT infection control committee therefore implemented a change of RPE for staff on "red" (COVID-19) wards from FRSMs to FFP3 respirators. In this study, we analyse the incidence of SARS-CoV-2 infection in HCWs before and after this transition.

\section{Results}

The total number of cases of SARS-CoV-2 infection amongst HCWs at CUHNFT increased throughout the study period, in keeping with the rising incidence of SARS-CoV-2 in the community (Figure 1 and Figure 1-source data 1 ). Similar proportions of cases were ascertained by symptomatic testing and asymptomatic screening on both green and red wards (Figure 1-figure supplement 1 ).

Prior to the change in RPE, cases per ward day were higher on red compared with green wards in seven out of eight weeks analysed ( $\mathrm{p}=0.016$, Wilcoxon signed-rank test, Figure 2 and Table 1 ). Following the change in RPE, the incidence of infection on red and green wards was similar, and not statistically different $(\mathrm{p}=0.5$, Wilcoxon signed-rank test, Figure 2 and Table 1 ). Strikingly, there was a strong positive correlation between the incidence of SARS-CoV-2 in the community and the number of cases per ward day on green $\left(\mathrm{R}^{2}=0.88\right)$ but not red wards $\left(\mathrm{R}^{2}=0.01\right)$ (Figure 2-figure supplement $\mathbf{1}$ ). Taken together, these results suggest that the majority of cases among HCWs on green wards were caused by community-acquired infection, whereas cases among HCWs on red wards were caused by both community-acquired infection and direct, ward-based infection from patients with COVID-19, effectively mitigated by the use of FFP3 respirators.

Figure 1: Comparison between total number of cases amongst HCWs and community incidence of SARS-CoV-2.

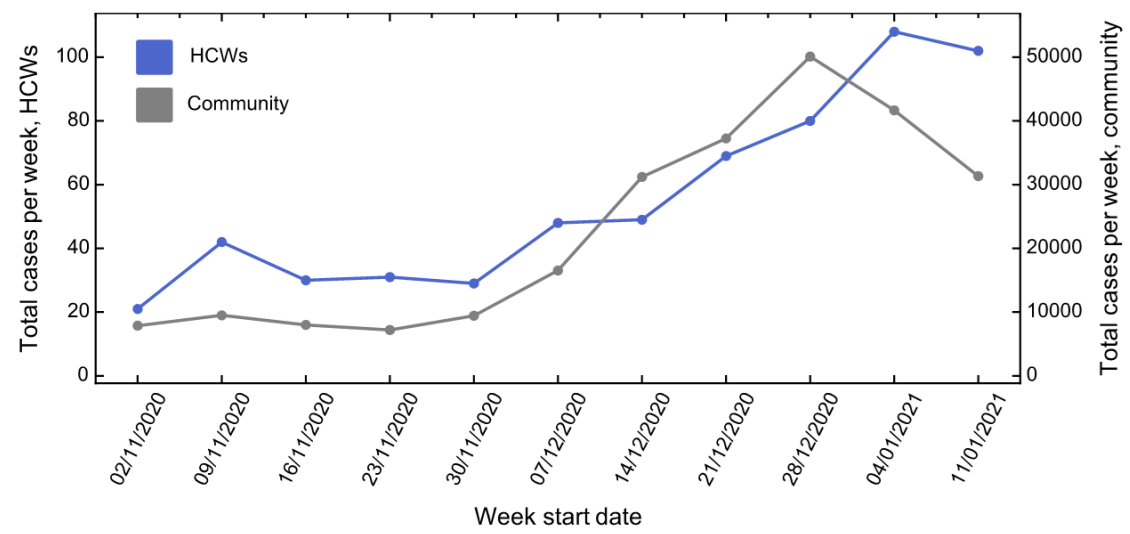

Community incidence is shown for the East of England, UK, derived from https://coronavirus.data.gov.uk/details/cases, with raw data shown in Figure 1-source data 1. 
Figure 2: Weekly cases per ward day amongst HCWs on red and green wards prior to and after the change in RPE.

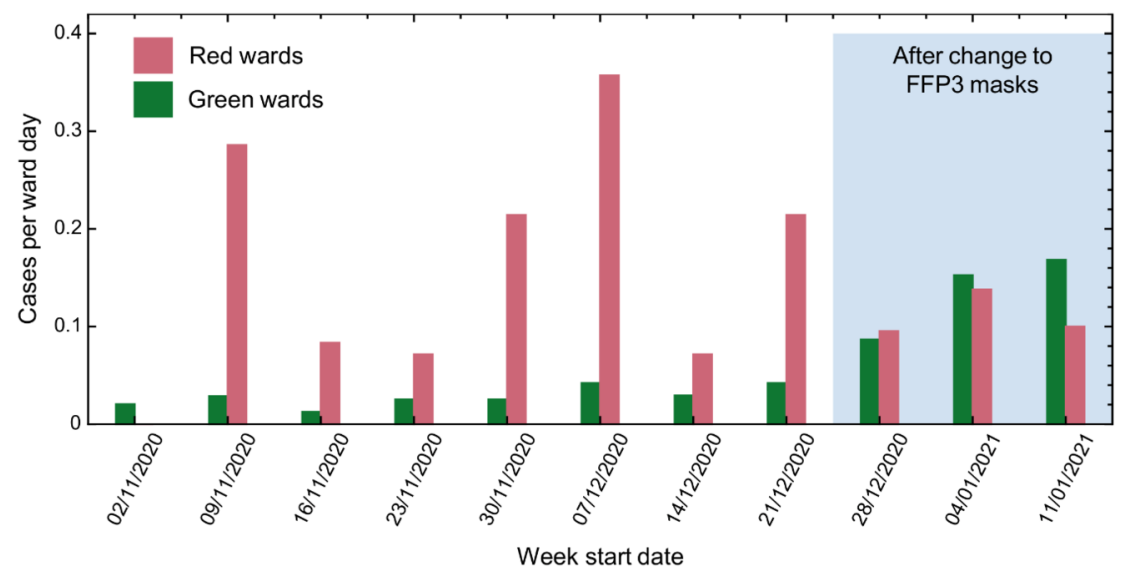

Table 1: Weekly numbers of cases amongst HCWs on red and green wards, and cases per ward day.

\begin{tabular}{llllllll}
\hline Week & Week start & Red cases & Red ward days & Red cases per ward day & Green cases & Green ward days & Green case \\
\hline 1 & $02 / 11 / 2020$ & 0 & 7 & 0 & 5 & 245 & 0.02 \\
2 & $09 / 11 / 2020$ & 2 & 7 & 0.286 & 7 & 245 & 0.028 \\
3 & $16 / 11 / 2020$ & 1 & 12 & 0.083 & 3 & 240 & 0.013 \\
4 & $23 / 11 / 2020$ & 1 & 14 & 0.071 & 6 & 238 & 0.025 \\
5 & $30 / 11 / 2020$ & 3 & 14 & 0.214 & 6 & 238 & 0.025 \\
6 & $07 / 12 / 2020$ & 5 & 14 & 0.357 & 10 & 238 & 0.042 \\
7 & $14 / 12 / 2020$ & 1 & 14 & 0.071 & 7 & 238 & 0.029 \\
8 & $21 / 12 / 2020$ & 3 & 14 & 0.214 & 10 & 238 & 0.042 \\
9 & $28 / 12 / 2020$ & 2 & 21 & 0.095 & 20 & 231 & 0.087 \\
10 & $04 / 01 / 2021$ & 4 & 29 & 0.138 & 34 & 223 & 0.152 \\
11 & $11 / 01 / 2021$ & 5 & 50 & 0.1 & 34 & 202 & 0.168 \\
\hline
\end{tabular}

Weeks following the change in RPE are highlighted in grey. Community incidence (total cases per week) is shown for the East of England, UK, with raw data shown in Figure 1-source data 1.

To further quantify the risk of infection for HCWs working on red and green wards, we generated a simple mathematical model. According to this model, the total risk of infection is divided into a risk from community-based exposure, and a risk from direct, ward-based exposure to patients. The risk from direct exposure on red wards was allowed to vary upon the introduction of FFP3 respirators, and was fitted to a maximum likelihood model. Inferred parameters and their confidence intervals are shown in Table 2. Our model produced a qualitatively close fit to the observed numbers of cases (Figures 3A-B ).

The inferred risk of direct infection from working on a green ward was low throughout the study period, and consistently lower than the risk of community-based exposure, which increased in proportion to rising levels of community incidence (Figure 3C ). By contrast, the risk of direct infection from working on a red ward before the change in RPE was considerably higher than the risk of community-based exposure, and approximately 47 -fold greater than the corresponding risk from working on a green ward (confidence interval 
[7.92, [?]]). Thus, whilst almost all cases on green wards were likely caused by infection in the community, cases on red wards at the beginning of the study period were attributed mainly to direct, ward-based exposure (Figures 3D-E ). Critically, our model further suggests that the introduction of FFP3 respirators provided $100 \%$ (confidence interval $[31.3 \%, 100 \%]$ ) protection against direct, ward-based COVID-19 infection (Table $\mathbf{2}, \mathrm{r} 2 / \mathrm{r} 1)$.

Figure 3: Mathematical modelling of the risks of infection for HCWs on red and green wards.
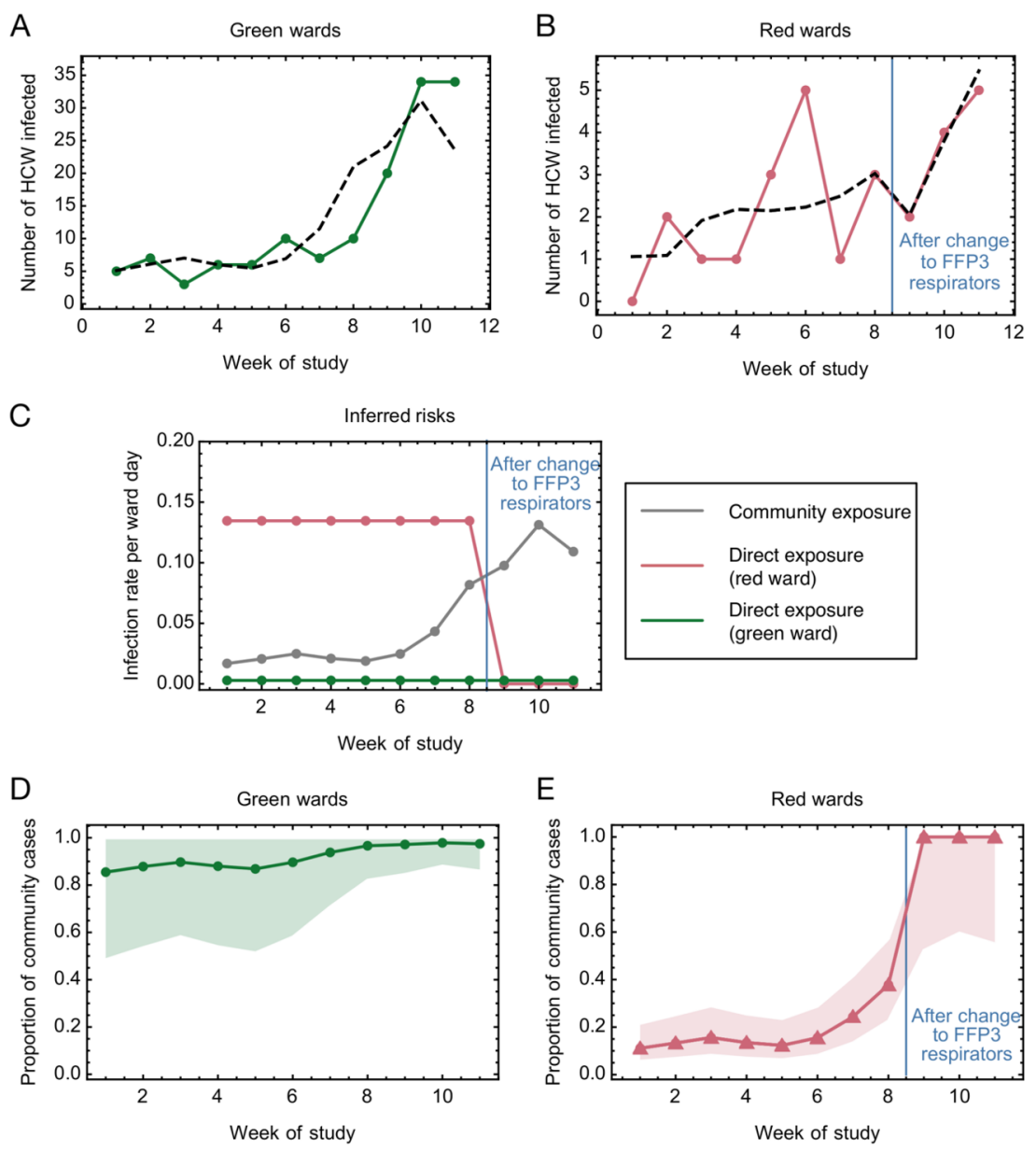

(A-B ) Comparison of modelled and actual cases. The model (black dashed line) aimed to reproduce the risks of infection among HCWs per ward day (A) on green wards (green solid line) and (B)on red wards (red solid line). (C) Risks inferred from the model. HCWs were vulnerable to COVID-19 infection from exposure to individuals in the community, with this risk increasing with community incidence (grey line). HCWs working on green wards faced a consistent, low risk of infection from direct, ward-based exposure (green line). HCWs working on red wards initially faced a much higher risk of infection from direct, ward-based exposure, falling to zero (confidence interval [0, 0.0804]) upon the introduction of FFP3 respirators. In this figure risks are expressed per ward day; a risk of 0.1 indicates that a particular source of risk would be expected to cause one HCW from the multiple staff present on a ward to develop an infection every 10 days that the ward was in operation. (D-E) Proportion of community-acquired cases. Proportion of infections on (D) green and (E ) red wards inferred to have arisen via exposure to individuals in the community (green line, green wards; red line, red wards; confidence intervals shaded). 
Table 2: Statistics and parameter ratios inferred from the model

\begin{tabular}{llll}
\hline Statistic & Model parameter & Maximum likelihood estimate & Confid \\
\hline Force of community-based infection per community case & $\mathrm{k}$ & $2.62 \times 10^{-6}$ & {$[1.97 \times$} \\
Force of direct infection per ward day (green ward) & $\mathrm{g}$ & $2.86 \times 10^{-3}$ & {$[0,1.43$} \\
Force of direct infection per ward day (red ward, pre-FFP3) & $\mathrm{r}_{1}$ & 0.135 & {$[0.0648$} \\
Force of direct infection per ward day (red ward, post-FFP3) & $\mathrm{r}_{2}$ & 0 & {$[0,0.08$} \\
Relative direct risk on red wards post- versus pre-FFP3 & $\mathrm{r}_{2} / \mathrm{r}_{1}$ & 0 & {$[0,0.68$} \\
Relative direct risk on red ward versus green ward pre-FFP3 & $\mathrm{r}_{1} / \mathrm{g}$ & 47.0 & {$[7.92,[$} \\
Relative direct risk on red ward versus green ward post-FFP3 & $\mathrm{r}_{2} / \mathrm{g}$ & 0 & {$[0,[?])$} \\
\hline
\end{tabular}

\section{Discussion}

HCWs may be exposed to SARS-CoV-2 from contacts in the community, from contacts with other HCWs, and from contacts with patients. In this study, we developed a mathematical model to evaluate the relative magnitudes of these risks, based on data collected during the second wave of the SARS-CoV-2 pandemic in the UK (Nov 2020-Jan 2021).

Whilst using FRSMs, the majority of infections among HCWs working on red wards could be attributed to direct exposure to patients with COVID-19. In contrast, the majority of infections among HCWs working on green wards arose from the community. After the change in RPE, cases attributed to ward-based exposure fell significantly, with FFP3 respirators providing 31-100\% protection (and most likely 100\%) against infection from patients with COVID-19. In keeping with previous observations [9-11], our findings therefore suggest that the use of FRSMs as RPE was insufficient to protect HCWs against infection from patients with COVID19. Conversely, excess infections amongst HCWs caring for patients with COVID-19 may be prevented by the use of FFP3 respirators, in combination with other PPE and infection control measures.

During the study period, the incidence of SARS-CoV-2 in England increased [17], with spread of the more transmissible B.1.1.7 variant [18]. By the ninth week of the study, $79 \%$ of cases in Cambridgeshire were caused by this variant [19]. Our observations on the use of FFP3 respirators (weeks 9-11) were therefore made at a time when the B.1.1.7 variant predominated, suggesting that they are robust to any associated increase in SARS-CoV-2 transmissibility in a hospital setting attributable to this variant.

Potential confounders of our observations, should they have changed over the course of the study, include: (a) rates of natural immunity amongst HCWs on red and green wards; however, the frequency of prior SARS-CoV-2 infections was low within CUHNFT [11]; (b) rates of vaccination of HCWs on red and green wards; however, the proportion of high-risk HCWs at CUHNFT offered vaccination prior to 08/01/21 was very low; (c) frequency of screening amongst HCWs on red and green wards; however, the proportion of cases ascertained by symptomatic testing and asymptomatic screening was similar in both settings; (d) compliance with infection control measures on red and green wards.

This observational study includes a small number of cases in a single Trust, and there may be alternative explanations for the different patterns of infection observed before and after the change in RPE. Nonetheless, our data highlight a need for further study into the appropriate level of RPE for HCWs caring for patients with COVID-19, as well as other respiratory viruses. In accordance with the precautionary principle, we propose a revision of RPE recommendations until more definitive information is available.

\section{Methods}

Study design and participants 
CUHNFT is a tertiary hospital in the UK with approximately 1,000 beds. During the pandemic, wards were categorised as "red", "amber" or "green". Patients with confirmed COVID-19 were cared for on red wards, and patients who had negative SARS-CoV-2 tests and no clinical features of COVID-19 on green wards. Patients awaiting test results, who had clinical features of COVID-19 but a negative test result, or who may have been exposed to SARS-CoV-2 were cared for on amber wards. RPE for staff on red wards was changed from FRSMs to FFP3 respirators on 22/12/20. HCWs on green wards continued to wear FRSMs. HCWs on all wards also wore eye protection.

A comprehensive PCR-based HCW screening programme is established at CUHNFT, with symptomatic testing offered as required and asymptomatic testing offered to all HCWs weekly [9, 12]. From 22/12/20, twice-weekly swabbing was offered on red wards and on wards where the most vulnerable patients were cared for. Cases were identified from a database of all positive results, which additionally encompasses positive results from community testing. This recorded the date of swab, onset of symptoms (if present) and in which clinical area the HCW worked.

The start of the study period was taken to be $02 / 11 / 20$, coinciding with an increase in community incidence of SARS-CoV-2 infection and formal implementation of weekly asymptomatic screening for all staff members. New infections on or prior to $27 / 12 / 20$ were attributed to exposure before the change in RPE. Infections detected later than this date were attributed to exposure after the change in RPE. This timing was chosen to reflect the median incubation period of SARS-CoV-2 (5.1 days), with $27 / 12 / 20$ falling five days after the change in RPE $[13,14]$. Since staff testing was not conducted at weekends, eight complete weeks were assessed in total prior to the change in RPE (Table 1).

A programme of SARS-CoV-2 vaccination using the BNT162b2 COVID-19 vaccine commenced at CUHNFT on $08 / 12 / 20$ [15]. In line with UK national guidance, the programme initially prioritised local residents over the age of 80. However, some HCWs who had been identified as at high risk from SARS-CoV-2 infection were also vaccinated, and were additionally prevented from working on red wards. From 08/01/21 the programme switched to vaccinating HCWs, with initial priority being given to staff on red wards. To avoid the potential for confounding, the end of the study period was therefore taken to be $15 / 01 / 21$, since minimal effect is expected in the first seven days after the first dose of vaccine [16].

Because of the rising number of admissions to CUHNFT with COVID-19, the number of red wards was increased from one at the beginning of November 2020 to seven by the week starting $11 / 01 / 21$. Six wards therefore changed from green to red during the period of data collection. Of 609 positive results over the entire study period, $169(27.8 \%)$ were included in this study. Exclusions encompassed HCWs who were not ward-based or worked between different wards with different red/amber/green status $(269 / 609,44.2 \%$ of positive results), HCW working on amber wards (9/609, 1.5\%), non-clinical staff (141/609, 23.1\%), and staff working in critical care areas $(21 / 609,3.5 \%)$, where different RPE was used throughout (Table 1 ).

If a staff member tested positive within five days of their ward changing colour, their case was classified according to the red/green status of their ward five days before their positive test (to allow for the incubation period, as above).

\section{General statistical analysis}

To account for changes in the number of red and green wards, weekly numbers of red or green ward days were calculated. Where $\mathrm{w}_{\mathrm{R}, \mathrm{d}}$ and $\mathrm{w}_{\mathrm{G}, \mathrm{d}}$ were the number of red and green wards open on day $\mathrm{d}$, the weekly numbers of ward days for week $\mathrm{i}$, denoted $\mathrm{W}_{\mathrm{R}, \mathrm{i}}$ and $\mathrm{W}_{\mathrm{G}, \mathrm{i}}$, were calculated as the sums of the number of each type of ward open on the days of that respective week.

$$
W_{G, i}=\sum_{d \in i} w_{G, d}
$$


and

$$
W_{R, i}=\sum_{d \in i} w_{R, d}
$$

The number of HCWs per ward was similar for red and green wards. For the purposes of comparison, we calculated the weekly number of cases amongst HCWs on red or green wards per red or green ward day, respectively (Table 1 ); we denote these weekly case numbers as $R_{i}$ and $G_{i}$. Wilcoxon's signed rank test was used to compare case rates between HCWs on red or green wards before and after the change of RPE (a non-parametric paired test).

Details of community incidence were calculated from publicly available data describing the East of England region of the UK (https://coronavirus.data.gov.uk/details/cases, data downloaded on 12/06/21), and were calculated as the sum of the number of cases reported in each week of the study. Raw data are shown in Figure 1-source data 1. Correlations between cases per ward day and community incidence were calculated using the Wolfram Mathematica software package, version 12.1.0.0.

\section{Mathematical modelling}

In order to quantify the effect of the change in RPE upon cases in red wards, we developed a mathematical model, considering the numbers of cases observed among HCWs as arising from a combination of wardspecific infection risks, which relate directly to working on a red or green ward, and non-ward-specific risks, which include infections arising from the community. We first wrote expressions for the infection risk facing workers in red and green wards on week i. For HCWs on green wards we write

$$
\lambda_{i}^{G}=\left(k C_{i-1}+g\right) W_{G, i}
$$

while for HCWs on red wards we write

$$
\lambda_{i}^{R}=\begin{array}{ll}
\left(k C_{i-1}+r_{1}\right) W_{R, i} & i<9 \\
\left(k C_{i-1}+r_{2}\right) W_{R, i} & i \geq 9
\end{array}
$$

Here the term $\mathrm{k}$ is a constant, while the value $\mathrm{C}_{\mathrm{i}-1}$ describes the number of observed cases in the local community in the previous week. Our use of data from the previous week reflects a generation time for SARS-CoV-2 of approximately seven days [20]; we assumed that HCWs diagnosed with COVID-19 infection during this study would have been infected by individuals who were diagnosed in the previous week. The model parameters $\mathrm{g}, \mathrm{r}_{1}$, and $\mathrm{r}_{2}$ describe ward-specific infection risks; FFP3 masks were used on red wards from week 9 onwards.

Model parameters were optimised using a likelihood framework, identifying the maximum value of the term; here the number of cases on each type of ward each week, $G_{i}$ and $R_{i}$, were represented as emissions from a Poisson distribution with parameter equal to the total risk of infection.

$$
L=\sum_{i}\left[\log \frac{\lambda_{i}^{G^{G} G_{i}}}{G_{i} !}+\log \frac{\lambda_{i}^{R^{R_{i}}}}{R_{i} !}\right]
$$

Confidence intervals for each parameter were then obtained using the likelihood function. Constrained likelihood optimisations were performed in which the likelihood was optimised subject to a fixed value of the parameter in question. Confidence intervals were defined as the region of parameter space in which the likelihood L was within 2 units of the maximum. Similarly, constrained optimisation was used to identify a confidence interval for parameter ratios such as $\mathrm{r}_{2} / \mathrm{r}_{1}$, which describes the relative risk to HCWs on red wards with, as opposed to prior to, the introduction of FFP3. 


\section{Ethics statement}

This study was conducted as a service evaluation of the CUHNFT staff testing services and PPE policy (CUHNFT clinical project ID3738). As a study of healthcare-associated infections, this investigation is exempt from requiring ethical approval under Section 251 of the NHS Act 2006 (see also the NHS Health Research Authority algorithm, available at http://www.hra-decision-tools.org.uk/research/, which concludes that no formal ethical approval is required).

\section{Acknowledgements}

This research was funded in part by a Wellcome Trust Senior Clinical Research Fellowship [108070/Z/15/Z] and grants from Addenbrooke's Charitable Trust and the NIHR Cambridge Biomedical Research Centre to MPW. NJM was funded by an MRC Clinician Scientist Fellowship [MR/P008801/1] and NHSBT workpackage [WPA15-02]. CJRI was supported by UKRI through the JUNIPER modelling consortium [grant number MR/V038613/1] and by the Medical Research Council [MC_UU_00002/11]. For the purpose of open access, the authors have applied a CC-BY public copyright licence to any author accepted manuscript version arising from this submission. We would like to thank everyone involved in the development and operation of the SARS-CoV-2 testing programme at CUH and the members of staff who have participated. We would also like to thank the Infection Control and Fit Testing teams.

\section{Competing interests}

The authors declare no competing interests.

\section{References}

[1] World Health Organization. Mask use in the context of COVID-19 Interim guidance, https://www.who.int/publications/i/item/advice-on-the-use-of-masks-in-the-community-during-homecare-and-in-healthcare-settings-in-the-context-of-the-novel-coronavirus-(2019-ncov)-outbreak [accessed $07 / 03 / 21]$

[2] Public Health England. COVID-19: Guidance for maintaining services within health and care settings Infection prevention and control recommendations, 2021, https://assets.publishing.service.gov.uk/government/uploads/system/uploads/attachment_data/file/954690/Infection_Prevention_and_Control_Guidance_January_2021.pdf [accessed 06/03/21].

[3] Public Health England. COVID-19: Guidance for maintaining services within health and care settings Infection prevention and control recommendations Version 1.2 2021 https://assets.publishing.service.gov.uk/government/uploads/system/uploads/attachment_data/file/990923/20210602_Infection_Prevention_and_Control_Guidance_for_maintaining_services_with_H_and_C_settings___1_.pdf [accessed 04/06/21]

[4] Centers for Disease Control and Prevention. Interim Infection Prevention and Control Recommendations for Healthcare Personnel During the Coronavirus Disease 2019 (COVID-19) Pandemic, https://www.cdc.gov/coronavirus/2019-ncov/hcp/infection-control-recommendations.html [accessed $07 / 04 / 21]$

[5] Public Health England. Independent High Risk AGP Panel systematic review: background paper, at https://assets.publishing.service.gov.uk/government/uploads/system/uploads/attachment_data/file/951192/Independent_High_Risk_AGP_Panel_systematic_review_background_paper.pdf [Accessed 07/03/21]

[6] Oksanen LM, Sanmark E, Oksanen S, Anttila VJ, Paterno JJ, Lappalainen M, Lehtonen L, Geneid A. Healthcare workers high COVID-19 infection rate: the source of infections and potential for respirators and surgical masks to reduce occupational infections. medRxiv . 2020 Jan 1.

[7] Ha JF. The COVID-19 pandemic, personal protective equipment and respirator: A narrative review. International Journal of Clinical Practice . 202074 : e13578. 
[8] Buising KL, Williamson D, Cowie BC, MacLachlan J, Orr E, MacIsaac C, Williams E, Bond K, Muhi S, McCarthy J, Maier AB, Irving L, Heinjus D, Kelly C, Marshall C. A hospital-wide response to multiple outbreaks of COVID-19 in health care workers: lessons learned from the field. The Medical Journal of Australia . 202015 .

[9] Rivett L, Sridhar S, Sparkes D, Routledge M, Jones NK, Forrest S, Young J, Pereira-Dias J, Hamilton WL, Ferris M, Torok ME, Meredith L; CITIID-NIHR COVID-19 BioResource Collaboration, Curran MD, Fuller S, Chaudhry A, Shaw A, Samworth RJ, Bradley JR, Dougan G, Smith KG, Lehner PJ, Matheson NJ, Wright G, Goodfellow IG, Baker S, Weekes MP. Screening of healthcare workers for SARS-CoV-2 highlights the role of asymptomatic carriage in COVID-19 transmission. Elife . 2020; 9:e58728.

[10] Eyre DW, Lumley SF, O'Donnell D, Campbell M, Sims E, Lawson E, Warren F, James T, Cox S, Howarth A, Doherty G, Hatch SB, Kavanagh J, Chau KK, Fowler PW, Swann J, Volk D, Yang-Turner F, Stoesser N, Matthews PC, Dudareva M, Davies T, Shaw RH, Peto L, Downs LO, Vogt A, Amini A, Young BC, Drennan PG, Mentzer AJ, Skelly DT, Karpe F, Neville MJ, Andersson M, Brent AJ, Jones N, Martins Ferreira L, Christott T, Marsden BD, Hoosdally S, Cornall R, Crook DW, Stuart DI, Screaton G; Oxford University Hospitals Staff Testing Group, Peto TE, Holthof B, O'Donnell AM, Ebner D, Conlon CP, Jeffery K, Walker TM. Differential occupational risks to healthcare workers from SARS-CoV-2 observed during a prospective observational study. Elife . 2020; 9 : e60675.

[11] Cooper DJ, Lear S, Watson L, Shaw A, Ferris M, Doffinger R, Bousfield R, Sharrocks K, Weekes MP, Warne B, Sparkes D, Jones NK, Rivett L, Routledge M, Chaudhry A, Dempsey K, Matson M, Lakha A, Gathercole G, O'Connor O, Wilson E, Shahzad O, Toms K, Thompson R, Halsall I, Halsall D, Houghton S, Papadia S, Kingston N, Stirrups KE, Graves B, Walker N, Stark H, CITIID-NIHR BioResource COVID-19 Collaboration Group, DeAngelis D, Seaman S, Dougan G, Bradley JR, Török ME, Goodfellow I, Baker S. A prospective study of risk factors associated with seroprevalence of SARS-CoV-2 antibodies in healthcare workers at a large UK teaching hospital. Available at SSRN: https://ssrn.com/abstract=3724855 [Accessed $06 / 03 / 21]$

[12] Jones NK, Rivett L, Sparkes D, Forrest S, Sridhar S, Young J, Pereira-Dias J, Cormie C, Gill H, Reynolds N, Wantoch M, Routledge M, Warne B, Levy J, Córdova Jiménez WD, Samad FNB, McNicholas C, Ferris M, Gray J, Gill M; CITIID-NIHR COVID-19 BioResource Collaboration, Curran MD, Fuller S, Chaudhry A, Shaw A, Bradley JR, Hannon GJ, Goodfellow IG, Dougan G, Smith KG, Lehner PJ, Wright G, Matheson NJ, Baker S, Weekes MP. Effective control of SARS-CoV-2 transmission between healthcare workers during a period of diminished community prevalence of COVID-19.eLife 2020;9:e59391.

[13] Lauer SA, Grantz KH, Bi Q, Jones FK, Zheng Q, Meredith HR, Azman AS, Reich NG, Lessler J. The incubation period of coronavirus disease 2019 (COVID-19) from publicly reported confirmed cases: estimation and application. Annals of Internal Medicine . 2020;172 : 577-82.

[14] McAloon C, Collins Á, Hunt K, Barber A, Byrne AW, Butler F, Casey M, Griffin J, Lane E, McEvoy D, Wall P, Green M, O'Grady L, More SJ. Incubation period of COVID-19: a rapid systematic review and meta-analysis of observational research. BMJ open . 2020;10 : e039652.

[15] Jones NK, Rivett L, Seaman S, Samworth RJ, Warne B, Workman C, Ferris M, Wright J, Quinnell N, Shaw A, Cambridge COVID-19 Collaboration, Goodfellow IG, Lehner PJ, Howes R, Wright G, Matheson NJ, Weekes MP. Single-dose BNT162b2 vaccine protects against asymptomatic SARS-CoV-2 infection. eLife 2021; 10: e68808 doi: 10.7554/eLife.68808

[16] Polack FP, Thomas SJ, Kitchin N, Absalon J, Gurtman A, Lockhart S, Perez JL, Pérez Marc G, Moreira ED, Zerbini C, Bailey R, Swanson KA, Roychoudhury S, Koury K, Li P, Kalina WV, Cooper D, Frenck RW Jr, Hammitt LL, Türeci Ö, Nell H, Schaefer A, Ünal S, Tresnan DB, Mather S, Dormitzer PR, Şahin U, Jansen KU, Gruber WC. Safety and efficacy of the BNT162b2 mRNA Covid-19 vaccine. New England Journal of Medicine. 2020 Dec 31;383(27):2603-15.

[17] Office of National Statistics. Coronavirus (COVID-19) Infection Survey, 
https://www.ons.gov.uk/peoplepopulationandcommunity/healthandsocialcare/conditionsanddiseases/bulletins/coronavirusC [accessed 07/04/21]

[18] Davies NG, Abbott S, Barnard RC, Jarvis CI, Kucharski AJ, Munday JD, Pearson AB, Russell TW, Tully DC, Washburne AD, Wenseleers T, Gimma A, Waites W, Wong KLM, van Zandvoort K, Silverman JD; CMMID COVID-19 Working Group; COVID-19 Genomics UK (COG-UK) Consortium, Diaz-Ordaz K, Keogh R, Eggo RM, Funk S, Jit M, Atkins KE, Edmunds WJ. Estimated transmissibility and impact of SARS-CoV-2 lineage B.1.1.7 in England. Science 2021; 372: eabg3055.

[19] https://coronavirus.data.gov.uk/details/cases

[20] Volz E, Mishra S, Chand M, Barrett JC, Johnson R, Geidelberg L, Hinsley WR, Laydon DJ, Dabrera G, O'Toole Á, Amato R, Ragonnet-Cronin M, Harrison I, Jackson B, Ariani CV, Boyd O, Loman N, McCrone JT, Gonçalves S, Jorgensen D, Myers R, Hill V, Jackson DK, Gaythorpe K, Groves N, Sillitoe J, Kwiatkowski DP, COG-UK, Flaxman S, Ratmann O, Bhatt S, Hopkins S, Gandy A, Rambaut A, Ferguson NM. Transmission of SARS-CoV-2 Lineage B. 1.1. 7 in England: Insights from linking epidemiological and genetic data. medRxiv. 2021:2020-12. doi: https://doi.org/10.1101/2020.12.30.20249034

Figure supplements

Figure 1-figure supplement 1. Proportion of cases ascertained by symptomatic testing and asymptomatic screening on green and red wards

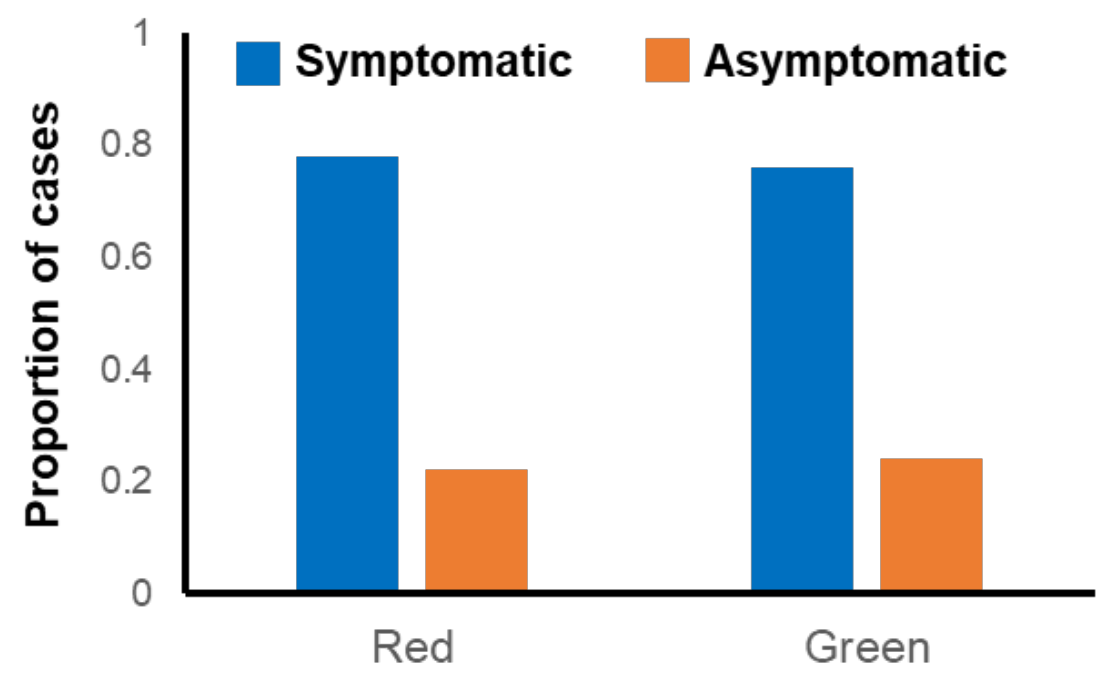

Figure 2-figure supplement 1. Relationships between cases per ward day and community incidence 

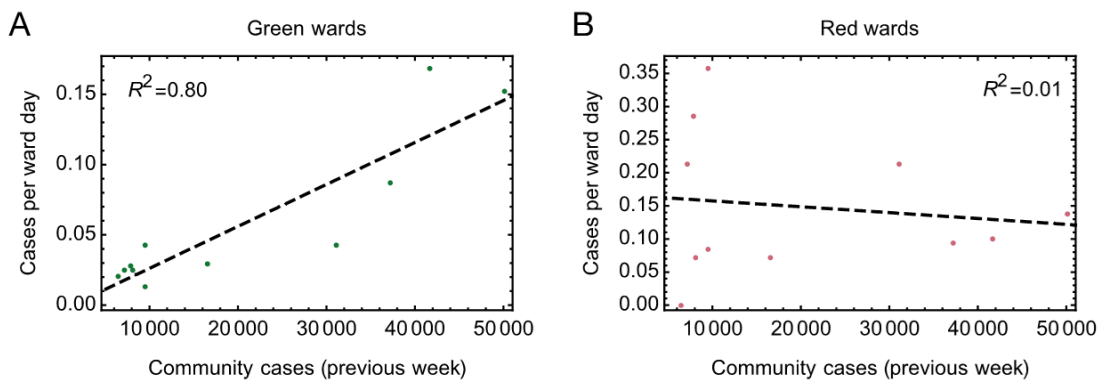

Cases per ward day amongst HCWs on green wards (A ) were strongly correlated with the number of community cases identified the previous week ( $\mathrm{p}$-value $<5 \times 10^{-5}$, Pearson correlation test), suggesting that infection in the community explains cases amongst HCWs on these wards. Conversely, cases per ward day amongst HCWs on red wards (B) did not correlate with the community incidence (p-value $>0.7$, Pearson correlation test). $\mathrm{R}^{2}$ values shown in the figures are coefficients of determination arising from linear regression calculations performed using the Mathematica software package (version 12.1.0.0).

\section{Source data}

Figure 1-source data 1. Raw case numbers for the East of England region during the period of study

\begin{tabular}{|c|c|c|c|c|c|}
\hline Area Type & Area Name & Area Code & Date & New cases by date & Weekly sum of cases \\
\hline region & East of England & E12000006 & $17 / 01 / 2021$ & 3011 & \\
\hline region & East of England & E12000006 & $16 / 01 / 2021$ & 3006 & \\
\hline region & East of England & E12000006 & $15 / 01 / 2021$ & 4471 & \\
\hline region & East of England & E12000006 & $14 / 01 / 2021$ & 4589 & 31341 \\
\hline region & East of England & E12000006 & $13 / 01 / 2021$ & 4907 & 32065 \\
\hline region & East of England & E12000006 & $12 / 01 / 2021$ & 5227 & 33124 \\
\hline region & East of England & E12000006 & $11 / 01 / 2021$ & 6130 & 33881 \\
\hline region & East of England & E12000006 & $10 / 01 / 2021$ & 3735 & 35093 \\
\hline region & East of England & E12000006 & $09 / 01 / 2021$ & 4065 & 36433 \\
\hline region & East of England & E12000006 & $08 / 01 / 2021$ & 5228 & 38441 \\
\hline region & East of England & E12000006 & $07 / 01 / 2021$ & 5801 & 41663 \\
\hline region & East of England & E12000006 & $06 / 01 / 2021$ & 6247 & 44429 \\
\hline region & East of England & E12000006 & $05 / 01 / 2021$ & 7235 & 47652 \\
\hline region & East of England & E12000006 & $04 / 01 / 2021$ & 9352 & 46446 \\
\hline region & East of England & E12000006 & 03/01/2021 & 6501 & 47120 \\
\hline region & East of England & E12000006 & $02 / 01 / 2021$ & 7288 & 49626 \\
\hline region & East of England & E12000006 & $01 / 01 / 2021$ & 4022 & 53511 \\
\hline region & East of England & E12000006 & $31 / 12 / 2020$ & 6475 & 50110 \\
\hline region & East of England & E12000006 & $30 / 12 / 2020$ & 8753 & 50158 \\
\hline region & East of England & E12000006 & $29 / 12 / 2020$ & 11120 & 48606 \\
\hline region & East of England & E12000006 & $28 / 12 / 2020$ & 5951 & 46320 \\
\hline region & East of England & E12000006 & $27 / 12 / 2020$ & 6549 & 44180 \\
\hline
\end{tabular}




\begin{tabular}{|c|c|c|c|c|c|}
\hline Area Type & Area Name & Area Code & Date & New cases by date & Weekly sum of cases \\
\hline region & East of England & E12000006 & $26 / 12 / 2020$ & 5736 & 41282 \\
\hline region & East of England & E12000006 & $25 / 12 / 2020$ & 1736 & 36518 \\
\hline region & East of England & E12000006 & $24 / 12 / 2020$ & 4335 & 37259 \\
\hline region & East of England & E12000006 & $23 / 12 / 2020$ & 5855 & 35301 \\
\hline region & East of England & E12000006 & $22 / 12 / 2020$ & 6356 & 32961 \\
\hline region & East of England & E12000006 & $21 / 12 / 2020$ & 6692 & 36360 \\
\hline region & East of England & E12000006 & $20 / 12 / 2020$ & 4591 & 36645 \\
\hline region & East of England & E12000006 & $19 / 12 / 2020$ & 3396 & 35505 \\
\hline region & East of England & E12000006 & $18 / 12 / 2020$ & 5135 & 33485 \\
\hline region & East of England & E12000006 & $17 / 12 / 2020$ & 4620 & 31219 \\
\hline region & East of England & E12000006 & $16 / 12 / 2020$ & 4715 & 29375 \\
\hline region & East of England & E12000006 & $15 / 12 / 2020$ & 4336 & 28224 \\
\hline region & East of England & E12000006 & $14 / 12 / 2020$ & 4426 & 25781 \\
\hline region & East of England & E12000006 & $13 / 12 / 2020$ & 2747 & 23475 \\
\hline region & East of England & E12000006 & $12 / 12 / 2020$ & 2245 & 21013 \\
\hline region & East of England & E12000006 & $11 / 12 / 2020$ & 2692 & 18765 \\
\hline region & East of England & E12000006 & $10 / 12 / 2020$ & 2314 & 16535 \\
\hline region & East of England & E12000006 & $09 / 12 / 2020$ & 2253 & 15089 \\
\hline region & East of England & E12000006 & $08 / 12 / 2020$ & 2088 & 14031 \\
\hline region & East of England & E12000006 & $07 / 12 / 2020$ & 2196 & 12718 \\
\hline region & East of England & E12000006 & $06 / 12 / 2020$ & 1301 & 11742 \\
\hline region & East of England & E12000006 & $05 / 12 / 2020$ & 1187 & 10927 \\
\hline region & East of England & E12000006 & $04 / 12 / 2020$ & 1379 & 10122 \\
\hline region & East of England & E12000006 & $03 / 12 / 2020$ & 1338 & 9441 \\
\hline region & East of England & E12000006 & $02 / 12 / 2020$ & 1438 & 8908 \\
\hline region & East of England & E12000006 & $01 / 12 / 2020$ & 1283 & 8528 \\
\hline region & East of England & E12000006 & $30 / 11 / 2020$ & 1515 & 8280 \\
\hline region & East of England & E12000006 & $29 / 11 / 2020$ & 768 & 7970 \\
\hline region & East of England & E12000006 & $28 / 11 / 2020$ & 807 & 7735 \\
\hline region & East of England & E12000006 & $27 / 11 / 2020$ & 1131 & 7481 \\
\hline region & East of England & E12000006 & $26 / 11 / 2020$ & 1028 & 7203 \\
\hline region & East of England & E12000006 & $25 / 11 / 2020$ & 1203 & 7238 \\
\hline region & East of England & E12000006 & $24 / 11 / 2020$ & 1029 & 7233 \\
\hline region & East of England & E12000006 & $23 / 11 / 2020$ & 1237 & 7158 \\
\hline region & East of England & E12000006 & $22 / 11 / 2020$ & 803 & 7307 \\
\hline region & East of England & E12000006 & $21 / 11 / 2020$ & 802 & 7354 \\
\hline region & East of England & E12000006 & $20 / 11 / 2020$ & 1056 & 7642 \\
\hline region & East of England & E12000006 & $19 / 11 / 2020$ & 1177 & 7998 \\
\hline region & East of England & E12000006 & $18 / 11 / 2020$ & 1250 & 8108 \\
\hline region & East of England & E12000006 & $17 / 11 / 2020$ & 1317 & 8289 \\
\hline region & East of England & E12000006 & $16 / 11 / 2020$ & 1593 & 8594 \\
\hline region & East of England & E12000006 & $15 / 11 / 2020$ & 913 & 8794 \\
\hline region & East of England & E12000006 & $14 / 11 / 2020$ & 983 & 9153 \\
\hline region & East of England & E12000006 & $13 / 11 / 2020$ & 1361 & 9293 \\
\hline region & East of England & E12000006 & $12 / 11 / 2020$ & 1377 & 9499 \\
\hline region & East of England & E12000006 & $11 / 11 / 2020$ & 1609 & 9666 \\
\hline region & East of England & E12000006 & $10 / 11 / 2020$ & 1457 & 9690 \\
\hline region & East of England & E12000006 & 09/11/2020 & 1799 & 9438 \\
\hline region & East of England & E12000006 & $08 / 11 / 2020$ & 1080 & 9176 \\
\hline region & East of England & E12000006 & $07 / 11 / 2020$ & 1007 & 8612 \\
\hline
\end{tabular}




\begin{tabular}{llllll}
\hline Area Type & Area Name & Area Code & Date & New cases by date & Weekly sum of cases \\
\hline region & East of England & E12000006 & $06 / 11 / 2020$ & 1109 & 8301 \\
region & East of England & E12000006 & $05 / 11 / 2020$ & 1115 & 7876 \\
region & East of England & E12000006 & $04 / 11 / 2020$ & 1045 & 7432 \\
region & East of England & E12000006 & $03 / 11 / 2020$ & 1146 & 7147 \\
region & East of England & E12000006 & $02 / 11 / 2020$ & 1374 & 7030 \\
region & East of England & E12000006 & $01 / 11 / 2020$ & 636 & 6889 \\
region & East of England & E12000006 & $31 / 10 / 2020$ & 722 & 6863 \\
region & East of England & E12000006 & $30 / 10 / 2020$ & 992 & 6653 \\
region & East of England & E12000006 & $29 / 10 / 2020$ & 974 & 6423 \\
region & East of England & E12000006 & $28 / 10 / 2020$ & 1019 & \\
region & East of England & E12000006 & $27 / 10 / 2020$ & 936 & \\
region & East of England & E12000006 & $26 / 10 / 2020$ & 1144 & \\
\hline
\end{tabular}

